\title{
Hermite-Hadamard's trapezoid and mid-point type inequalities on a disk
}

\author{
M. Rostamian Delavar ${ }^{\text {* }}$ (D, S.S. Dragomir ${ }^{2}$ and M. De La Sen ${ }^{3}$
}

${ }^{*}$ Correspondence:
m.rostamian@ub.ac.ir
${ }^{1}$ Department of Mathematics,
Faculty of Basic Sciences, University
of Bojnord, Bojnord, Iran
Full list of author information is
available at the end of the article

available at the end of the article

\begin{abstract}
Some trapezoid and mid-point type inequalities related to the Hermite-Hadamard inequality on the disk of center $C=(a, b)$ and radius $R, D(C, R) \subseteq \mathbb{R}^{2}$, are investigated. It is shown that the estimated value obtained in the trapezoid and mid-point type inequalities has a relation with the integral of the partial derivative of the considered function on $\partial(C, R)$, the boundary of $D(C, R)$.
\end{abstract}

MSC: 26D15;26A51;26D07

Keywords: Hermite-Hadamard inequality; Convex functions of double variable; Trapezoid and mid-point type inequalities

\section{Introduction}

Let $f: I \subseteq \mathbb{R} \rightarrow \mathbb{R}$ be a convex mapping defined on the interval $I$ of real numbers and $a, b \in I$ with $a<b$. The following double inequality

$$
f\left(\frac{a+b}{2}\right)(b-a) \leq \int_{a}^{b} f(x) d x \leq(b-a) \frac{f(a)+f(b)}{2},
$$

is known in the literature as Hermite-Hadamard inequality for convex mappings. For more results and generalization about (1), see $[1,5-11]$ and the references therein.

An interesting problem in (1) is estimating the difference between the right term and the integral of $f$ on $[a, b]$ and also estimating the difference between the left term and the integral of $f$ on $[a, b]$.

In [3], the authors have obtained an estimation for the difference between the right term of (1) and the integral of $f$ as follows.

Theorem 1.1 Let $f: I^{\circ} \subseteq \mathbb{R} \rightarrow \mathbb{R}$ be a differentiable mapping on $I^{\circ}, a, b \in I^{\circ}$ with $a<b$. If $\left|f^{\prime}\right|$ is convex on $[a, b]$, then the following inequality holds:

$$
\left|\int_{a}^{b} f(x) d x-(b-a) \frac{f(a)+f(b)}{2}\right| \leq \frac{1}{8}(b-a)^{2}\left(\left|f^{\prime}(a)\right|+\left|f^{\prime}(b)\right|\right) .
$$

As we can see in Theorem 1.1, the estimation value is in connection with the absolute value of the derivative of the considered function on the boundary points of the corresponding interval $[a, b]$. In fact the striped area shown in Fig. 1, which is equivalent to the

(c) The Author(s) 2019. This article is distributed under the terms of the Creative Commons Attribution 4.0 International License (http://creativecommons.org/licenses/by/4.0/), which permits unrestricted use, distribution, and reproduction in any medium, provided you give appropriate credit to the original author(s) and the source, provide a link to the Creative Commons license, and indicate if changes were made. 
Figure 1 Trapezoid type inequality

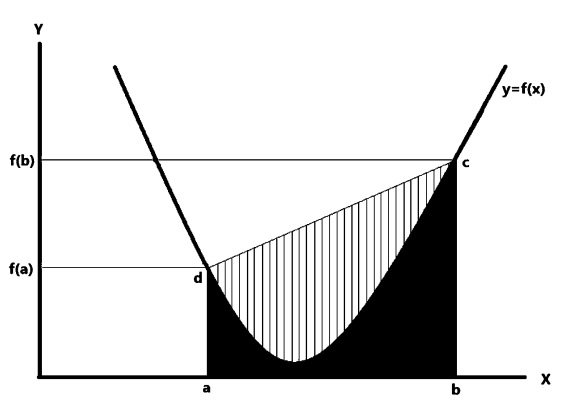

Figure 2 Mid-point type inequality

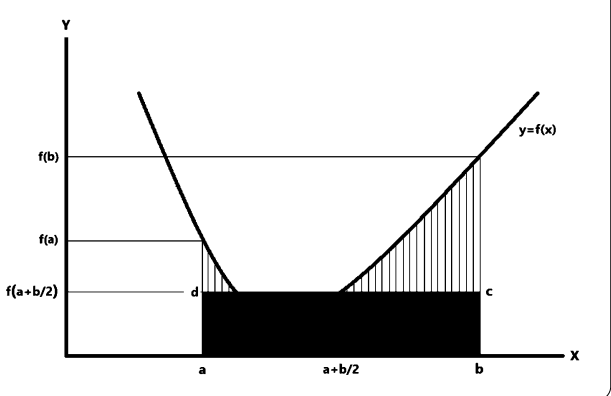

difference between the area of trapezoid $a b c d$ and the area under the graph of $f$, is estimated in (2) as well. Due to this geometric property, we call inequality (2) trapezoid type inequalities related to the Hermite-Hadamard inequality.

Also in [4], the author obtained an estimation for the difference between the left term of (1) and the integral of $f$ :

Theorem $1.2([4])$ Let $f: I^{\circ} \subseteq \mathbb{R} \rightarrow \mathbb{R}$ be a differentiable mapping on $I^{\circ}, a, b \in I^{\circ}$ with $a<b$. If $\left|f^{\prime}\right|$ is convex on $[a, b]$, then we have

$$
\left|\int_{a}^{b} f(x) d x-(b-a) f\left(\frac{a+b}{2}\right)\right| \leq \frac{1}{8}(b-a)^{2}\left(\left|f^{\prime}(a)\right|+\left|f^{\prime}(b)\right|\right) .
$$

According to (3), the striped area shown in Fig. 2, which is in fact equivalent to the difference between the area under the graph of $f$ and the area of rectangle $a b c d$, is estimated. Due to this geometric property, we call inequality (3) mid-point type inequalities related to the Hermite-Hadamard inequality.

Now let us consider a point $C=(a, b) \in \mathbb{R}^{2}$ and the disk $D(C, R)$ centered at the point $C$ and having the radius $R>0$. The following inequality has been obtained in [2], which is a Hermite-Hadamard inequality related to convex functions defined on the disk $D(C, R)$ in $\mathbb{R}^{2}$.

Theorem 1.3 If the mapping $f: D(C, R) \rightarrow \mathbb{R}$ is convex on $D(C, R)$, then one has the inequality

$$
f(C) \leq \frac{1}{\pi R^{2}} \iint_{D(C, R)} f(x, y) d x d y \leq \frac{1}{2 \pi R} \int_{\partial(C, R)} f(\gamma) d l(\gamma),
$$


where $\partial(C, R)$ is the circle centered at the point $C=(a, b)$ with radius $R$. The above inequalities are sharp.

Motivated by the above-mentioned works, we investigate the trapezoid and mid-point type inequalities related to (4). We show that on a disk $D(C, R)$, these kinds of estimations have a relation with the integral of $\left|\frac{\partial f}{\partial r}\right|$ (in polar coordinates) on $\partial(C, R)$, the boundary of the disk $D(C, R)$, provided that $\left|\frac{\partial f}{\partial r}\right|$ is convex with respect to the variable $r \in[0, R]$.

\section{Main results}

The first result of this section is the trapezoid type inequality related to (4).

Theorem 2.1 Consider a set $I \subset \mathbb{R}^{2}$ with $D(C, R) \subset I^{\circ}$. Suppose that the mapping $f$ : $D(C, R) \rightarrow \mathbb{R}$ has continuous partial derivatives in the disk $D(C, R)$ with respect to the variables $r$ and $\theta$ in polar coordinates. If, for any constant $\theta \in[0,2 \pi]$, the function $\left|\frac{\partial f}{\partial r}\right|$ is convex with respect to the variable $r$ on $[0, R]$, then

$$
\left|\frac{1}{2 \pi R} \int_{\partial(C, R)} f(\gamma) d l(\gamma)-\frac{1}{\pi R^{2}} \iint_{D(C, R)} f(x, y) d x d y\right| \leq \frac{1}{6 \pi} \int_{\partial(C, R)}\left|\frac{\partial f}{\partial r}\right|(\gamma) d l(\gamma) .
$$

Proof For a constant $\theta \in[0,2 \pi]$, if we consider

$$
x(r)=a+r \cos \theta
$$

and

$$
y(r)=b+r \sin \theta,
$$

then we have $\left([\dot{x}(r)]^{2}+[\dot{y}(r)]^{2}\right)^{\frac{1}{2}}=\left(\sin ^{2}(\theta)+\cos ^{2}(\theta)\right)^{\frac{1}{2}}=1$, where $\dot{x}, \dot{y}$ are the derivatives of $x, y$, respectively, with respect to the variable $r$ on $[0, R]$. So, by the use of integration by parts, we have the following equalities:

$$
\begin{aligned}
\int_{0}^{R} & \frac{\partial f}{\partial r}(a+r \cos \theta, b+r \sin \theta) r^{2} d r=\left.r^{2} f(a+r \cos \theta, b+r \sin \theta)\right|_{0} ^{R} \\
& -2 \int_{0}^{R} f(a+r \cos \theta, b+r \sin \theta) r d r=R^{2} f(a+R \cos \theta, b+R \sin \theta) \\
& -2 \int_{0}^{R} f(a+r \cos \theta, b+r \sin \theta) r d r .
\end{aligned}
$$

The integration of (6) with respect to $\theta$ on $[0,2 \pi]$ implies that

$$
\begin{aligned}
& R^{2} \int_{0}^{2 \pi} f(a+R \cos \theta, b+R \sin \theta) d \theta-2 \int_{0}^{2 \pi} \int_{0}^{R} f(a+r \cos \theta, b+r \sin \theta) r d r d \theta \\
& \quad=\int_{0}^{2 \pi} \int_{0}^{R} \frac{\partial f}{\partial r}(a+r \cos \theta, b+r \sin \theta) r^{2} d r d \theta .
\end{aligned}
$$


Since $\left|\frac{\partial f}{\partial r}\right|$ is convex with respect to the variable $r$ on $[0, R]$ for any $\theta \in[0,2 \pi]$, then

$$
\begin{aligned}
\mid R^{2} & \int_{0}^{2 \pi} f(a+R \cos \theta, b+R \sin \theta) d \theta-2 \int_{0}^{2 \pi} \int_{0}^{R} f(a+r \cos \theta, b+r \sin \theta) r d r d \theta \mid \\
\leq & \int_{0}^{2 \pi} \int_{0}^{R}\left|\frac{\partial f}{\partial r}\right|(a+r \cos \theta, b+r \sin \theta) r^{2} d r d \theta \\
= & \int_{0}^{2 \pi} \int_{0}^{R}\left|\frac{\partial f}{\partial r}\right|\left(\frac{r}{R}(a+R \cos \theta, b+R \sin \theta)+\left(1-\frac{r}{R}\right)(a, b)\right) r^{2} d r d \theta \\
\leq & \int_{0}^{2 \pi} \int_{0}^{R} \frac{r^{3}}{R}\left|\frac{\partial f}{\partial r}\right|(a+R \cos \theta, b+R \sin \theta) d r d \theta \\
& +\int_{0}^{2 \pi} \int_{0}^{R} r^{2}\left(1-\frac{r}{R}\right)\left|\frac{\partial f}{\partial r}\right|(C) d r d \theta \\
= & \frac{R^{3}}{4} \int_{0}^{2 \pi}\left|\frac{\partial f}{\partial r}\right|(a+R \cos \theta, b+R \sin \theta) d \theta+\frac{\pi R^{3}}{6}\left|\frac{\partial f}{\partial r}\right|(C) .
\end{aligned}
$$

Now, consider the curve $\gamma:[0,2 \pi] \rightarrow \mathbb{R}^{2}$ given by

$$
\gamma:\left\{\begin{array}{l}
x(\theta)=a+R \cos \theta, \\
y(\theta)=b+R \sin \theta,
\end{array} \quad \theta \in[0,2 \pi] .\right.
$$

Then $\gamma([0,2 \pi])=\partial(C, R)$, and we write (integrating with respect to arc length)

$$
\begin{aligned}
\int_{\partial(C, R)}\left|\frac{\partial f}{\partial r}\right|(\gamma) d l(\gamma) & =\int_{0}^{2 \pi}\left|\frac{\partial f}{\partial r}\right|(x(\theta), y(\theta))\left([\dot{x}(\theta)]^{2}+[\dot{y}(\theta)]^{2}\right)^{\frac{1}{2}} d \theta \\
& =R \int_{0}^{2 \pi}\left|\frac{\partial f}{\partial r}\right|(a+R \cos \theta, b+R \sin \theta) d \theta .
\end{aligned}
$$

From (7) and (8) we obtain

$$
\begin{aligned}
& \left|R^{2} \int_{0}^{2 \pi} f(a+R \cos \theta, b+R \sin \theta) d \theta-2 \int_{0}^{2 \pi} \int_{0}^{R} f(a+r \cos \theta, b+r \sin \theta) r d r d \theta\right| \\
& \quad \leq \frac{R^{2}}{4} \int_{\partial(C, R)}\left|\frac{\partial f}{\partial r}\right|(\gamma) d l(\gamma)+\frac{\pi R^{3}}{6}\left|\frac{\partial f}{\partial r}\right|(C) .
\end{aligned}
$$

Also using the convexity of $\left|\frac{\partial f}{\partial r}\right|$ in (4) we have

$$
\begin{aligned}
\left|\frac{\partial f}{\partial r}\right|(C) & \leq \frac{1}{\pi R^{2}} \int_{0}^{2 \pi} \int_{0}^{R}\left|\frac{\partial f}{\partial r}\right|(a+r \cos \theta, b+r \sin \theta) d r d \theta \\
& \leq \frac{1}{2 \pi R} \int_{\partial(C, R)}\left|\frac{\partial f}{\partial r}\right|(\gamma) d l(\gamma) .
\end{aligned}
$$

So by replacing (10) in (9) we obtain

$$
\begin{aligned}
& \left|R \int_{\partial(C, R)} f(\gamma) d l(\gamma)-2 \int_{0}^{2 \pi} \int_{0}^{R} f(a+r \cos \theta, b+r \sin \theta) r d r d \theta\right| \\
& \quad \leq \frac{R^{2}}{3} \int_{\partial(C, R)}\left|\frac{\partial f}{\partial r}\right|(\gamma) d l(\gamma) .
\end{aligned}
$$


Finally dividing (11) with $2 \pi R^{2}$ we get

$$
\left|\frac{1}{2 \pi R} \int_{\partial(C, R)} f(\gamma) d l(\gamma)-\frac{1}{\pi R^{2}} \iint_{D(C, R)} f(x, y) d x d y\right| \leq \frac{1}{6 \pi} \int_{\partial(C, R)}\left|\frac{\partial f}{\partial r}\right|(\gamma) d l(\gamma) .
$$

Example 2.2 Consider the bifunction $f(x, y)=R-\sqrt{(x-a)^{2}+(y-b)^{2}}$ defined on the disk $D(C, R)$. In polar coordinates we have that

$$
f(a+r \cos \theta, b+r \sin \theta)=R-r
$$

for $0 \leq r \leq R, \theta \in[0,2 \pi]$ and specially $f(a+R \cos \theta, b+R \sin \theta)=0$ for all $\theta \in[0,2 \pi]$. So

$$
\begin{aligned}
& \left|\frac{1}{2 \pi R} \int_{\partial(C, R)} f(\gamma) d l(\gamma)-\frac{1}{\pi R^{2}} \iint_{D(C, R)} f(x, y) d x d y\right| \\
& \quad=\frac{1}{\pi R^{2}} \iint_{D(C, R)} f(x, y) d x d y=\frac{1}{\pi R^{2}} \int_{0}^{2 \pi} \int_{0}^{R}(R-r) r d r d \theta=\frac{R}{3} .
\end{aligned}
$$

On the other hand, it is not hard to see that $\left|\frac{\partial f}{\partial r}\right|(a+R \cos \theta, b+R \sin \theta)=1$ for all $\theta \in[0,2 \pi]$, and so

$$
\frac{1}{6 \pi} \int_{\partial(C, R)}\left|\frac{\partial f}{\partial r}\right|(\gamma) d l(\gamma)=\frac{R}{3} .
$$

Then identities (12) and (13) show that inequality (5) is sharp.

The following result is the mid-point type inequality related to (4).

Theorem 2.3 Consider a set $I \subset \mathbb{R}^{2}$ with $D(C, R) \subset I^{\circ}$. Suppose that the mapping $f$ : $D(C, R) \rightarrow \mathbb{R}$ has continuous partial derivatives in the disk $D(C, R)$ with respect to the variables $r$ and $\theta$ in polar coordinates. If, for any constant $\theta \in[0,2 \pi]$, the function $\left|\frac{\partial f}{\partial r}\right|$ is convex with respect to the variable $r$ on $[0, R]$, then

$$
\left|\frac{1}{\pi R^{2}} \iint_{D(C, R)} f(x, y) d x d y-f(C)\right| \leq \frac{2}{3 \pi} \int_{\partial(C, R)}\left|\frac{\partial f}{\partial r}\right|(\gamma) d l(\gamma) .
$$

Proof As we have seen in the proof of Theorem 2.1, for a constant $\theta \in[0,2 \pi]$, if we consider $x(r)=a+r \cos \theta$ and $y(r)=b+r \sin \theta$, then we have $\left([\dot{x}(r)]^{2}+[\dot{y}(r)]^{2}\right)^{\frac{1}{2}}=1$. So from fundamental theorem of calculus we have

$$
\int_{0}^{R} \frac{\partial f}{\partial r}(a+r \cos \theta, b+r \sin \theta) d r=f(a+R \cos \theta, b+R \sin \theta)-f(C) .
$$

Hence

$$
\begin{aligned}
\int_{0}^{2 \pi} & \int_{0}^{R} \frac{\partial f}{\partial r}(a+r \cos \theta, b+r \sin \theta) d r d \theta \\
\quad= & \int_{0}^{2 \pi} f(a+R \cos \theta, b+R \sin \theta) d \theta-2 \pi f(C)
\end{aligned}
$$


which implies that

$$
\int_{0}^{2 \pi} \int_{0}^{R} \frac{\partial f}{\partial r}(a+r \cos \theta, b+r \sin \theta) d r d \theta=\frac{1}{R} \int_{\partial(C, R)} f(\gamma) d l(\gamma)-2 \pi f(C) .
$$

Now from (15) we obtain

$$
\begin{aligned}
& \left|\frac{1}{2 \pi R} \int_{\partial(C, R)} f(\gamma) d l(\gamma)-f(C)\right| \\
& \quad \leq \frac{1}{2 \pi} \int_{0}^{2 \pi} \int_{0}^{R}\left|\frac{\partial f}{\partial r}\right|(a+r \cos \theta, b+r \sin \theta) d r d \theta .
\end{aligned}
$$

Since $\left|\frac{\partial f}{\partial r}\right|$ is convex, then it follows that

$$
\begin{aligned}
\left|\frac{1}{2 \pi R} \int_{\partial(C, R)} f(\gamma) d l(\gamma)-f(a, b)\right| \\
\leq \frac{1}{2 \pi}\left[\int_{0}^{2 \pi} \int_{0}^{R}\left|\frac{\partial f}{\partial r}\right|\left(\frac{r}{R}(a+R \cos \theta, b+R \sin \theta)+\left(1-\frac{r}{R}\right)(a, b)\right) d r d \theta\right] \\
\leq \frac{1}{2 \pi}\left[\int_{0}^{2 \pi} \int_{0}^{R} \frac{r}{R}\left|\frac{\partial f}{\partial r}\right|(a+R \cos \theta, b+R \sin \theta) d r d \theta\right. \\
\left.\quad+\int_{0}^{2 \pi} \int_{0}^{R}\left(1-\frac{r}{R}\right)\left|\frac{\partial f}{\partial r}\right|(C) d r d \theta\right] \\
=\frac{1}{4 \pi} \int_{\partial(C, R)}\left|\frac{\partial f}{\partial r}\right|(\gamma) d l(\gamma)+\frac{R}{2}\left|\frac{\partial f}{\partial r}\right|(C) .
\end{aligned}
$$

From the triangle inequality and (16) we get

$$
\begin{aligned}
\left|\frac{1}{\pi R^{2}} \int_{0}^{2 \pi} \int_{0}^{R} f(a+r \cos \theta, b+r \sin \theta) d r d \theta-f(C)\right| \\
\leq \frac{1}{4 \pi} \int_{\partial(C, R)}\left|\frac{\partial f}{\partial r}\right|(\gamma) d l(\gamma)+\frac{R}{2}\left|\frac{\partial f}{\partial r}\right|(C) \\
\quad+\left|\frac{1}{\pi R^{2}} \int_{0}^{2 \pi} \int_{0}^{R} f(a+r \cos \theta, b+r \sin \theta) r d r d \theta-\frac{1}{2 \pi R} \int_{\partial(C, R)} f(\gamma) d l(\gamma)\right| .
\end{aligned}
$$

Since $\left|\frac{\partial f}{\partial r}\right|$ satisfies the Hermite-Hadamard inequality (4), then

$$
\left|\frac{\partial f}{\partial r}\right|(C) \leq \frac{1}{2 \pi R} \int_{\partial(C, R)}\left|\frac{\partial f}{\partial r}\right|(\gamma) d l(\gamma) .
$$

So, by replacing (5) and the inequality in (17) above, we obtain

$$
\left|\frac{1}{\pi R^{2}} \iint_{D(C, R)} f(x, y) d x d y-f(C)\right| \leq \frac{2}{3 \pi} \int_{\partial(C, R)}\left|\frac{\partial f}{\partial r}\right|(\gamma) d l(\gamma) .
$$

Remark 2.4 If the functions $f$ and $\left|\frac{\partial f}{\partial r}\right|$ are convex on $D(C, R)$, then by the use of inequalities (5), (14), and (4) we have

$$
0 \leq \frac{1}{\pi R^{2}} \iint_{D(C, R)} f(x, y) d x d y-f(C) \leq \frac{2}{3 \pi} \int_{\partial(C, R)}\left|\frac{\partial f}{\partial r}\right|(\gamma) d l(\gamma)
$$



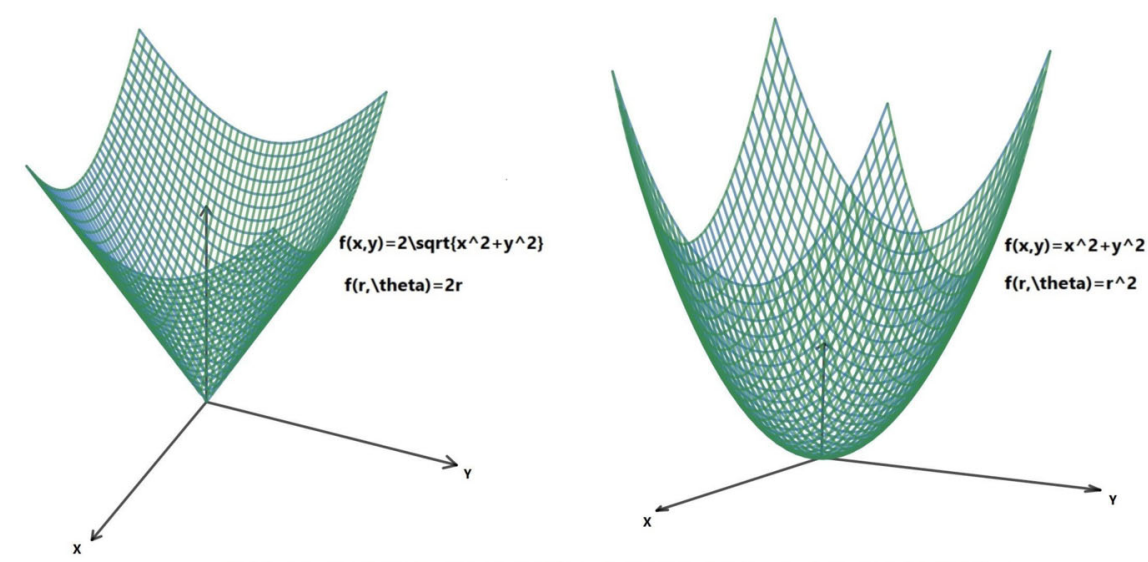

Figure 3 Comparison between the graph of $f$ and the graph of it's partial derivative with respect to the variable $r$

and

$$
0 \leq \frac{1}{2 \pi R} \int_{\partial(C, R)} f(\gamma) d l(\gamma)-\frac{1}{\pi R^{2}} \iint_{D(C, R)} f(x, y) d x d y \leq \frac{1}{6 \pi} \int_{\partial(C, R)}\left|\frac{\partial f}{\partial r}\right|(\gamma) d l(\gamma)
$$

Example 2.5 There exists a function satisfying all the conditions of Remark 2.4 as well. Consider the function $f(x, y)=x^{2}+y^{2}$ with $(x, y) \in \mathbb{R}^{2}$ defined on a disk $D((0,0), R)$. It is clear that $f(r, \theta)=r^{2}$ and $\left|\frac{\partial f}{\partial r}\right|=2 r$, which is equivalent to $f(x, y)=2 \sqrt{x^{2}+y^{2}}$ with $(x, y) \in \mathbb{R}^{2}$ defined on a disk $D((0,0), R)$. As we can see in Fig. 3, the functions $f$ and $\left|\frac{\partial f}{\partial r}\right|$ are convex.

\section{Acknowledgements}

Author M. De La Sen is grateful to the Spanish Government for funding received from the European Fund of Regional Development FEDER through Grant DPI2015-64766-R and to UPV/EHU for Grant PGC 17/33.

Funding

Not applicable.

Availability of data and materials

Not applicable.

\section{Competing interests}

The authors declare that they have no competing interests.

Authors' contributions

All authors read and approved the final manuscript.

\section{Author details}

${ }^{1}$ Department of Mathematics, Faculty of Basic Sciences, University of Bojnord, Bojnord, Iran. ${ }^{2}$ Mathematics, College of Engineering \& Science, Victoria University, Melbourne City, Australia. ${ }^{3}$ Institute of Research and Development of Processes, University of Basque Country, Bilbao, Spain.

\section{Publisher's Note}

Springer Nature remains neutral with regard to jurisdictional claims in published maps and institutional affiliations.

Received: 26 December 2018 Accepted: 8 April 2019 Published online: 18 April 2019

\section{References}

1. Alomari, M., Darus, M., Kirmaci, U.S.: Refinements of Hadamard-type inequalities for quasi-convex functions with applications to trapezoidal formula and to special means. Comput. Math. Appl. 59, 225-232 (2010)

2. Dragomir, S.S.: On Hadamard's inequality on a disk. J. Inequal. Pure Appl. Math. 1(1), Article 2 (2000)

3. Dragomir, S.S., Agarwal, R.P.: Two inequalities for differentiable mappings and applications to special means of real numbers and to trapezoidal formula. Appl. Math. Lett. 11, 91-95 (1998) 
4. Kirmaci, U.S.: Inequalities for differentiable mappings and applications to special means of real numbers and to midpoint formula. Appl. Math. Comput. 147(1), 137-146 (2004)

5. Pearce, C.E.M., Pecaric, J.E.: Inequalities for differentiable mappings with application to special means and quadrature formula. Appl. Math. Lett. 13,51-55 (2000)

6. Rostamian Delavar, M., De La Sen, M.: Some generalizations of Hermite-Hadamard type inequalities. SpringerPlus 5 1661 (2016)

7. Rostamian Delavar, M., Dragomir, S.S.: On $\eta$-convexity. Math. Inequal. Appl. 20, 203-216 (2017)

8. Sarikaya, M.Z., Saglam, A., Yildirim, H.: On some Hadamard-type inequalities for $h$-convex functions. J. Math. Inequal. 2, 335-341 (2008)

9. Wasowicz, S., Witkowski, A.: On some inequality of Hermite-Hadamard type. Opusc. Math. 32(3), 591-600 (2012)

10. Yang, G.S.: Inequalities of Hadamard type for Lipschitzian mappings. J. Math. Anal. Appl. 260, 230-238 (2001)

11. Yang, G.S., Hwang, D.Y., Tseng, K.L.: Some inequalities for differentiable convex and concave mappings. Comput. Math. Appl. 47, 207-216 (2004)

Submit your manuscript to a SpringerOpen ${ }^{\circ}$ journal and benefit from:

- Convenient online submission

Rigorous peer review

Open access: articles freely available online

- High visibility within the field

- Retaining the copyright to your article

Submit your next manuscript at $\gg$ springeropen.com 